\title{
OPEN The comparison of pancreatic and hepatic steatosis in healthy liver donor candidates
}

\author{
Bedriye Koyuncu Sokmen ${ }^{1 凶}$, Tolga Sahin ${ }^{2}$, Alihan Oral ${ }^{3}$, Erdem Kocak ${ }^{2}$ \& Nagihan Inan ${ }^{1}$
}

The aim of this study was to investigate the relationship between nonalcoholic fatty liver disease (NAFLD) and pancreatic steatosis (PS) in patients with biopsy-proven NAFLD. 228 patients with biopsy-proven NAFLD patients who admitted to the Faculty of Medicine of Demiroglu Bilim University between 2004 and 2019 were included in the study. Demographic, laboratory, histological and radiological findings of the patients were recorded retrospectively. Hepatosteatosis (HS) levels were measured by both CT and biopsy, while PS levels were measured by 3 different CT-based techniques. $89(39 \%)$ of the patients were female and $139(61 \%)$ were male. The mean body mass index (BMI) was $27.2 \pm 4.0$. Biochemical parameters were within normal limits. Liver biopsy showed a significant correlation with HS grade on CT scan $(\mathrm{p}<0.001)$. When CT findings were compared, a significant correlation was found between PS and HS $(p<0.05)$, but there was no correlation between the HS level in biopsy and the pancreatic adiposity on $C T(p>0.05)$. Our study was the first to compare biopsyproven NAFLD and PS, and no correlation was found between biopsy-proven NAFLD and PS.

The increasing frequency of obesity is one of the most important health problems in modern medicine. The prevalence of obesity has doubled in the last three decades since the $1980 \mathrm{~s}$. A total of 1.9 billion adults were overweight in 2014, and 600 million of these individuals were obese. Currently, obesity and obesity-related disorders such as diabetes mellitus (DM), metabolic syndrome (MetS)) and nonalcoholic fatty liver disease (NAFLD) have become important global health problems ${ }^{1,2}$. MetS was first described by Raevan in 1988 as Syndrome X. It includes obesity, hyperglycaemia, dyslipidaemia, hypertension and insulin resistance (IR). NAFLD is strongly associated with obesity and is also accepted as a hepatic manifestation of metabolic syndrome. NAFLD has become the most common liver disease in developed countries due to increasing obesity, changing eating habits and sedentary lifestyle ${ }^{3}$. Nonalcoholic steatohepatitis (NASH) develops in $30 \%$ to $40 \%$ of patients with NAFLD. NASH is defined as an advanced, chronic form of NAFLD, and liver cirrhosis can develop in $10-30 \%$ of patients with $\mathrm{NASH}^{4}$. The increased prevalence of obesity and DM and recent advances in the treatment of chronic viral hepatitis have made NAFLD one of the leading causes of liver cirrhosis worldwide ${ }^{5}$.

The rise in obesity and NAFLD incidence has led to increased attention on ectopic pancreatic fat accumulation, especially in the last two decades. Pancreatic steatosis (PS) was first described by Ogilvie in 1933 in obese individuals. He used 'pancreatic lipomatosis' as a term for fatty infiltration in the pancreas ${ }^{6}$. Since this initial description, pancreatic fat deposition has been studied by many researchers in different studies, and many different definitions and nomenclatures have been used, such as pancreatic lipomatosis, lipomatous pseudohypertrophy, nonalcoholic fatty pancreatic disease (NAFPD) and nonalcoholic fatty steatopancreatitis (NASP) ${ }^{7-9}$. Recently, NAFPD has become the most widely used term in the literature for the description of pancreatic fat accumulation.

Pancreatic fat is associated with systemic vascular complications. It is particularly associated with subclinical atherosclerosis and increases the prevalence of diabetic retinopathy ${ }^{10}$. Kim et al. showed that fatty pancreatic infiltration is associated with a higher risk of carotid atherosclerosis in non-obese patients with type $2 \mathrm{DM}^{11}$. Pancreatic steatosis indirect evidence with respect to the relationship acute and chronic pancreatitis. Given the toxic effect of fat on acinar cells, exocrine pancreatic insufficiency could occur in the evolution of pancreatic steatosis, at least theoretically. Fatty infiltration of the pancreas has been proven to be a significant risk factor for pancreatic fistula formation Obesity is a well-known risk factor for PC and there are some preliminary data to support an association between FP and pancreatic cancer too ${ }^{12}$.

${ }^{1}$ Department of Radiology, Faculty of Medicine, Demiroglu Bilim University, Büyükdere Street. No: 120 Esentepe - Sisli, 34394 Istanbul, Turkey. ${ }^{2}$ Department of Gastroenterology, Demiroglu Bilim University, Istanbul, Turkey. ${ }^{3}$ Department of Internal Medicine, Demiroglu Bilim University, Istanbul, Turkey. ${ }^{\square}$ email: bedriyekoyuncu@ yahoo.com 


\begin{tabular}{|c|c|c|}
\hline & Mean \pm SD & Min-max \\
\hline Age & $34.3 \pm 8.7$ & $19-57$ \\
\hline \multicolumn{3}{|l|}{ Gender } \\
\hline Female & $89(39.0)$ & \\
\hline Male & $139(61.0)$ & \\
\hline Height $(\mathrm{cm})$ & $169.1 \pm 9.6$ & $140-197$ \\
\hline Weight (kg) & $78.0 \pm 12.6$ & $44-112$ \\
\hline $\mathrm{BMI}\left(\mathrm{kg} / \mathrm{m}^{2}\right)$ & $27.2 \pm 4.0$ & $18-37.8$ \\
\hline $\mathrm{Hb}(\mathrm{g} / \mathrm{dL})$ & $14.4 \pm 1.6$ & $9.9-18.1$ \\
\hline WBC $\left(10^{3} / \mathrm{UL}\right)$ & $7.15 \pm 1.91$ & $3.36-12$ \\
\hline $\operatorname{PLT}\left(10^{3} / \mathrm{UL}\right)$ & $248.6 \pm 63.4$ & $135-622$ \\
\hline INR & $1.04 \pm 0.09$ & $0.8-1.6$ \\
\hline AST (IU/L) & $18.5 \pm 5.7$ & $10-41$ \\
\hline ALT (IU/L) & $22.8 \pm 14.1$ & $3-103$ \\
\hline ALP (IU/L) & $72.8 \pm 24.4$ & $6-242$ \\
\hline GGT (IU/L) & $20.8 \pm 15.7$ & $3-111$ \\
\hline Albumin $(\mathrm{g} / \mathrm{dL})$ & $4.68 \pm 0.32$ & $3.7-5.5$ \\
\hline T.biluribin (mg/dL) & $0.59 \pm 0.31$ & $0.1-2.5$ \\
\hline Bun (mg/dL) & $12.2 \pm 3.1$ & $5-25$ \\
\hline Creatinin $(\mathrm{mg} / \mathrm{dL})$ & $0.79 \pm 0.16$ & $0.4-1.3$ \\
\hline $\mathrm{Na}(\mathrm{miliEq} / \mathrm{L})$ & $140.1 \pm 2.2$ & $135-146$ \\
\hline $\mathrm{K}(\mathrm{miliEq} / \mathrm{L})$ & $4.4 \pm 0.3$ & $3.5-5.5$ \\
\hline $\mathrm{FPG}(\mathrm{mg} / \mathrm{dL})$ & $95.2 \pm 9.5$ & $71-168$ \\
\hline Insulin $(\mu \mathrm{IU} / \mathrm{mL})$ & $11.1 \pm 6.2$ & $1.21-45.9$ \\
\hline HbAlc (\%) & $5.3 \pm 0.5$ & $2.7-10$ \\
\hline HOMA-IR & $2.60 \pm 1.61$ & $0.25-13.6$ \\
\hline T. Cholesterol (mg/dl) & $186.0 \pm 41.4$ & 90-304 \\
\hline TGL (mg/dl) & $118.0 \pm 66.1$ & $10-487$ \\
\hline $\mathrm{TSH}(\mu \mathrm{IU} / \mathrm{mL})$ & $2.00 \pm 1.21$ & $0.23-8.65$ \\
\hline FT3 (pmol/L) & $4.83 \pm 1.56$ & $1.2-23$ \\
\hline FT4 (pmol/L) & $13.7 \pm 10.3$ & $0.99-138$ \\
\hline
\end{tabular}

Table 1. Laboratory findings and general demographic characteristics of study population. BMI Body mass index, $\mathrm{cm}$ centimeter, $\mathrm{kg}$ kilogram, $\mathrm{Hb}$ Hemoglobin, $W B C$ white blood cell, PLT platelet, INR international normalized ratio, AST aspartate aminotransferase, $A L T$ alanine aminotransferase, GGT gama glutamyl transpeptidase, $A L P$ alkaline phosphatase, $N a$ sodium, $K$ potassium, $F P G$ fasting plasma glucose, $H b A 1 c$ Hemoglobin A1c, HOMA-IR Insulin resistance, TGL tryglyserid, TSH thyroid stimulating hormone, FT3 free T3, FT4 free T4.

In the study by Bi et al., a total of 13 studies involving 49329 subjects were included. This analyses elucidated the presence of non alcoholic fatty pancreas disease (NAFPD) and was associated with a significant increased risk of metabolic syndrome, hypertension, nonalcoholic fatty liver disease (NAFLD), diabetes mellitus and central obesity. The association between NAFPD and hyperlipidaemia was not statistically significant ${ }^{13}$. As a result, NAFPD is associated with metabolic syndrome.

Many radiological methods have been reported for the evaluation of PS in the literature, such as ultrasound (US), endoscopic ultrasound (EUS), computed tomography (CT) and magnetic resonance imaging (MRI). CT is one of the most commonly used radiologic methods for the evaluation of PS and NAFLD ${ }^{14}$. The power of CT in the evaluation of pancreatic adiposity has been demonstrated in many studies ${ }^{15}$. The efficacy of CT in measuring hepatosteatosis (HS) is more pronounced. CT has become an alternative method for liver biopsy in many transplantation centres due to its strength in the determination of HS in liver transplant donor candidates ${ }^{16}$. Despite the power of CT and many other radiological methods in the diagnosis of HS, liver biopsy is still considered the gold standard method in diagnosing NAFLD ${ }^{17}$. Although many studies have associated NAFLD and NAFPD using different methods, there are no studies comparing NAFPD with biopsy-proven NAFLD. The main aim of this study was to investigate the relationship between NAFPD and NAFLD in patients with biopsy-proven NAFLD.

\section{Results}

A total of 228 patients were included in the study. Of these patients, 89 were female (39\%), and 139 were male (61\%). The mean age for the study population was $34.3 \pm 8.7$. The mean height was $169.1 \pm 9.6 \mathrm{~cm}$, and the mean weight was $78.0 \pm 12.6 \mathrm{~kg}$. The mean BMI was $27.2 \pm 4.0$. Table 1 shows the general characteristics of the study population. 


\begin{tabular}{|c|c|c|c|c|c|}
\hline & \multicolumn{5}{|c|}{ BMI } \\
\hline & \multicolumn{2}{|c|}{ No } & \multicolumn{2}{|c|}{ Yes $(>25)$} & \multirow[b]{2}{*}{$\mathbf{p}^{*}$} \\
\hline & $\mathbf{n}$ & $\%$ & $\mathbf{n}$ & $\%$ & \\
\hline \multicolumn{6}{|c|}{ Pancreas mean steatosis grade } \\
\hline Grade 1 steatosis & 69 & 88.5 & 102 & 68.0 & 0.003 \\
\hline Grade 2 steatosis & 8 & 10.3 & 39 & 26.0 & \\
\hline Grade 3 steatosis & 1 & 1.3 & 9 & 6.0 & \\
\hline \multicolumn{6}{|c|}{ P-S steatosis grade } \\
\hline Grade 1 steatosis & 69 & 88.5 & 103 & 68.7 & 0.004 \\
\hline Grade 2 steatosis & 8 & 10.3 & 38 & 25.3 & \\
\hline Grade 3 steatosis & 1 & 1.3 & 9 & 6.0 & \\
\hline \multicolumn{6}{|c|}{$\mathrm{P} / \mathrm{S}$ steatosis grade } \\
\hline Grade 1 steatosis & 69 & 88.5 & 103 & 68.7 & 0.004 \\
\hline Grade 2 steatosis & 8 & 10.3 & 38 & 25.3 & \\
\hline Grade 3 steatosis & 1 & 1.3 & 9 & 6.0 & \\
\hline
\end{tabular}

Table 2. The relationship between pancreatic steatosis and BMI in 3 different CT methods. ${ }^{*}$ Spearman correlation.

\begin{tabular}{|l|l|l|l|}
\hline \multicolumn{4}{|l|}{ Liver steatosis on CT } \\
\hline & No $(\mathbf{n}=\mathbf{8 3})$ & Yes $(\mathbf{n}=\mathbf{1 4 5})$ & $\mathbf{p}^{*}$ \\
\hline Spearman correlation $^{*}$ & $1.18 \pm 0.41$ & $1.35 \pm 0.59$ & 0.025 \\
\hline Spearman correlation $^{*}$ & $1.18 \pm 0.41$ & $1.35 \pm 0.59$ & 0.032 \\
\hline Spearman correlation $^{*}$ & $1.18 \pm 0.41$ & $1.35 \pm 0.59$ & 0.032 \\
\hline
\end{tabular}

Table 3. Comparison of HS and PS for different CT scan technics. * Spearman correlation.

\begin{tabular}{|l|l|l|}
\hline \multicolumn{3}{|l|}{ Percentile degree of hepatic steatosis (\%) } \\
\hline & $\mathbf{r}$ & $\mathbf{p}^{*}$ \\
\hline Pancreas mean steatosis grade & 0.149 & 0.023 \\
\hline P-S mean steatosis grade & 0.142 & 0.032 \\
\hline P/S mean steatosis grade & 0.142 & 0.032 \\
\hline
\end{tabular}

Table 4. Correlation analysis for pancreatic steatosis grade for each CT measument model. ${ }^{\star}$ Spearman correlation.

The complete blood count and the other biochemical laboratory findings of the study population were generally within the normal range. Laboratory findings of the study population were correlated with healthy individuals. Table 1 summarizes the average laboratory findings for the study patients.

BMI and PS levels for each CT measurement method were compared. PS was significantly positively correlated with BMI (Table 2). When patients were compared according to HS on CT, PS was significantly correlated with HS according to CT measurements (Table 3 ).

Patients were evaluated according to the degree of pancreatic and hepatic steatosis on CT scans. The degree of pancreatic adiposity was significantly correlated with the percentile degree of HS in the CT scan (Table 4). However, there was no correlation between the level of HS in liver biopsy and the degree of PS on CT scan $(\mathrm{p}>0.05)$ (Table 5).

Spearman correlation analysis was performed for PS in the last step. While BMI was significantly correlated with PS, age and laboratory findings were not correlated with PS in the study population $(p>0.05)($ Table 6$)$.

\section{Discussion}

Many studies have evaluated the relationship between NAFLD and NAFPD by different radiological methods, but no clinical studies have used liver biopsy. Several studies have found a positive correlation between NAFPD and NAFLD using different radiological methods ${ }^{18-21}$, but none of them include liver biopsy, which is considered the gold standard method for the diagnosis of HS. To the best of our knowledge, this is the first study to compare pancreatic adiposity with NAFLD diagnosed by liver biopsy. In our study, although pancreatic and hepatic steatosis rates measured by CT were correlated as in many other studies, no significant correlation was found between liver biopsy findings and PS. In addition, a significant correlation was found between BMI and 


\begin{tabular}{|l|r|r|r|r|r|}
\hline & \multicolumn{5}{|l|}{ Hepatosteatosis in biopsy } \\
\cline { 2 - 5 } & No & \multicolumn{1}{l|}{ Yes } & \\
\cline { 2 - 5 } & n & \multicolumn{1}{l|}{$\%$} & \multicolumn{1}{l|}{ n } & \multicolumn{1}{l|}{$\%$} & \multicolumn{1}{l}{ p } \\
\hline Pancreas mean steatosis grade \\
\hline Grade 1 steatosis & 71 & 74.7 & 100 & 75.2 & 0.692 \\
\hline Grade 2 steatosis & 21 & 22.1 & 26 & 19.5 & \\
\hline Grade 3 steatosis & 3 & 3.2 & 7 & 5.3 & \\
\hline Grade 1 steatosis & 72 & 75.8 & 100 & 75.2 & 0.732 \\
\hline P-S mean steatosis grade \\
\hline Grade 2 steatosis & 20 & 21.1 & 26 & 19.5 & \\
\hline Grade 3 steatosis & 3 & 3.2 & 7 & 5.3 & \\
\hline P/S mean steatosis grade \\
\hline Grade 1 steatosis & 72 & 75.8 & 100 & 75.2 & 0.732 \\
\hline Grade 2 steatosis & 20 & 21.1 & 26 & 19.5 & \\
\hline Grade 3 steatosis & 3 & 3.2 & 7 & 5.3 & \\
\hline
\end{tabular}

Table 5. The relationship between HS in biopsy and pancreatic steatosis in CT scan.

\begin{tabular}{|c|c|c|c|c|c|c|}
\hline \multicolumn{3}{|c|}{ Pancreas mean steatosis grade } & \multicolumn{2}{|c|}{\begin{tabular}{|l|} 
P-S mean \\
steatosis grade
\end{tabular}} & \multicolumn{2}{|c|}{\begin{tabular}{|l|} 
P/S mean \\
steatosis grade
\end{tabular}} \\
\hline & rho & $\mathbf{p}$ & rho & $\mathbf{p}$ & rho & $\mathbf{p}$ \\
\hline Age & 0.127 & 0.055 & 0.128 & 0.053 & 0.128 & 0.053 \\
\hline BMI $\left(\mathrm{kg} / \mathrm{m}^{2}\right)$ & 0.195 & 0.003 & 0.200 & 0.002 & 0.200 & 0.002 \\
\hline AST (IU/L) & -0.010 & 0.880 & 0.006 & 0.931 & 0.006 & 0.931 \\
\hline ALT (IU/L) & 0.002 & 0.980 & 0.009 & 0.889 & 0.009 & 0.889 \\
\hline ALP (IU/L) & 0.031 & 0.641 & 0.020 & 0.767 & 0.020 & 0.767 \\
\hline GGT (IU/L) & 0.082 & 0.217 & 0.086 & 0.197 & 0.086 & 0.197 \\
\hline Albumin(g/dL) & -0.039 & 0.563 & -0.053 & 0.430 & -0.053 & 0.430 \\
\hline T.Bil (mg/dL) & -0.161 & 0.065 & -0.155 & 0.079 & -0.155 & 0.079 \\
\hline FPG $(\mathrm{mg} / \mathrm{dL})$ & 0.119 & 0.074 & 0.107 & 0.109 & 0.107 & 0.109 \\
\hline Insulin $(\mu \mathrm{IU} / \mathrm{mL})$ & 0.124 & 0.061 & 0.113 & 0.088 & 0.113 & 0.088 \\
\hline $\operatorname{HbAlc}(\%)$ & 0.021 & 0.754 & 0.013 & 0.848 & 0.013 & 0.848 \\
\hline HOMA-IR & 0.126 & 0.057 & 0.114 & 0.087 & 0.114 & 0.087 \\
\hline Total cholesterol(mg/dL) & 0.033 & 0.623 & 0.036 & 0.590 & 0.036 & 0.590 \\
\hline TGL (mg/dL) & 0.040 & 0.547 & 0.035 & 0.597 & 0.035 & 0.597 \\
\hline $\mathrm{TSH}(\mu \mathrm{IU} / \mathrm{mL})$ & -0.053 & 0.423 & -0.046 & 0.485 & -0.046 & 0.485 \\
\hline FT3 (pmol/L) & 0.003 & 0.969 & 0.004 & 0.954 & 0.004 & 0.954 \\
\hline FT4 (pmol/L) & 0.052 & 0.433 & 0.051 & 0.447 & 0.051 & 0.447 \\
\hline
\end{tabular}

Table 6. Correlation analysis between demographic, laboratory data and pancreatic steatosis in study. Spearman correlation analysis.

pancreatic fat levels. The levels of PS were not correlated with the ages or laboratory parameters of the patients in our study. Our study population consisted of only healthy individuals, and we think that the composition of the study population may be the main cause of these results.

Since the pancreas and liver tissues originate from the same endoderm embryologically, ectopic fat accumulation in both organs can be expected. In many studies, the relationship between NAFPD, NAFLD and obesity led many researchers to assume that NAFPD and NAFLD develop due to similar aetiologies. However, recent data show that different molecular mechanisms mediate the pathophysiology and natural history of pancreatic and hepatic steatosis. In addition, liver fat deposition develops through intracellular lipid accumulation in hepatocytes, while fat accumulation in the pancreatic tissue occurs with intercellular adipocyte infiltration in both acinar and islet cells in the interlobular region ${ }^{22,23}$.

The current literature shows that pancreatic tissue is more sensitive to fat infiltration than liver tissue ${ }^{24}$. Although our study population was not composed of obese individuals, a significant positive correlation was found between the degree of pancreatic fat and mean BMI value in our study. We think that this finding reflects both the accuracy and sensitivity of the methods used in our study and confirms the current literature knowledge.

PS has been evaluated in many studies using different radiological methods, such as US, EUS, CT and MRI, and most of these studies are of East Asian origin ${ }^{10,18,19,25-29}$. US can be a simple, inexpensive and widely accessible 
radiological technique for the detection of PS, but it also has some disadvantages. Pancreatic visualization may not be possible with US in obese patients. Pancreatic fibrosis may be a misleading factor at diagnosis because pancreatic fibrosis shows a hyperechogenic appearance similar to fat accumulation in pancreatic tissue on US. Pancreatic echogenicity is usually compared with liver echogenicity on US, but the liver is a highly metabolically active organ, and its echogenicity can vary continuously. This may lead to different and misleading results in the diagnosis of PS with US ${ }^{6,30}$. The main advantage of EUS is the proximity of the ultrasound probe to the pancreas, which enables higher resolution imaging of pancreatic tissue compared to CT and MRI, but EUS is an invasive procedure and has some disadvantages, such as a high complication risk and sedation requirement. The fact that both transabdominal US and EUS are operator-dependent methods can lead to different outcomes and may cause diagnostic errors ${ }^{14}$. All of these factors make the reliability of studies using US-based methods controversial. To date, many MRI-based radiological techniques have been used to measure PS ${ }^{27-29}$. MRI is a noninvasive and safe radiological method, but different outcomes were obtained in different MRI-based studies in patients with NAFLD and NAFPD. In some MR-based studies, there was a significant positive correlation between HS and $\mathrm{PS}^{31-33}$, whereas in some MR-based studies, there was no correlation ${ }^{34}$. Due to these different results in different studies, the power and adequacy of MRI to detect the relationship between NAFLD and NAFPD is controversial. $\mathrm{CT}$ is considered one of the most powerful methods in the detection of PS ${ }^{14,15}$.

In many radiological studies, it was found that there was a correlation between the level of pancreatic adiposity measured by CT and histopathological examination of pancreatic tissue ${ }^{15,35}$. In a previous study the corrected value of pancreatic CT attenuation based on splenic attenuation was compared with the histologic pancreatic fat fraction in 62 patients who underwent any type of pancreatic resection. The histologic pancreatic fat fraction was correlated with the P-S $(r=-0.616, p<0.01)$ and $\mathrm{P} / \mathrm{S}(\mathrm{r}=-0.622, \mathrm{p}<0.01)$. Based on the above research results, we investigated the relationship between hepatic and pancreatic steatosis parameters measured by nonenhanced $\mathrm{CT}^{15}$. CT is also known to be correlated with liver biopsy for the detection of HS in many studies ${ }^{16}$. Since CT is one of the most highly correlated methods with liver and pancreas biopsies, CT was chosen as the reference radiological method in our study.

Radiological studies have shown different degrees of steatosis in different anatomical regions of the pancreas ${ }^{36}$. The development of the pancreas from two separate buds, ventral and dorsal, in the embryological period and differences in this development period have been suggested to be the major cause of this condition ${ }^{22,36}$. In this study, to prevent possible measurement errors due to the non-diffuse distribution of pancreatic adiposity in the parenchyma, the level of pancreatic fat was measured from 3 different anatomical regions of the pancreas using 3 different measurement techniques.

Although pancreatic and hepatic steatosis appear to be similar diseases associated with obesity and MetS, the pathophysiological mechanisms associated with the development of both diseases are different ${ }^{24}$. When studies on NAFPD in the literature are examined, it is seen that the mean BMI of the patient population in our study is similar to that in other studies ${ }^{13}$. Nonetheless, no significant correlation was found between liver biopsy findings and the degree of pancreatic fat on CT. Since the degree of liver steatosis was determined by liver biopsy, which is accepted as the gold standard method, we believe that the data obtained in our study have higher accuracy and reliability than other studies in the literature. The use of liver biopsy as a reference diagnostic method for NAFLD, non-diffuse distribution of pancreatic adiposity, differences in study populations (ethnicity, number of patients), and the use of different radiological methods and measurement techniques may be the main reasons for the different results between our study and other studies. The results of our study showed that although NAFLD and NAFPD appear to be similar diseases, they are not exactly the same disease, do not have the same pathophysiological mechanisms and do not have a simultaneous prognosis.

Our study had some limiting factors, such as the retrospective design and absence of pancreatic biopsy. However, pancreatic biopsy is a highly invasive procedure and does not seem to be useful due to the high risk of complications and ethical concerns. In addition, since ectopic fat accumulation does not show homogeneous distribution in the pancreatic tissue, different and misleading clinical results may be obtained depending on the site of the pancreatic biopsy.

\section{Conclusion}

The relationship between NAFLD and NAFPD is controversial in the literature. Our study is the first to include liver biopsy to study this issue. We concluded that liver biopsy has not been included in the studies so far, and the absence of liver biopsy may be the main cause of different results in studies investigating the relationship between NAFLD and NAFPD. More accurate data can be obtained with studies involving biopsy-proven NAFLD cases.

\section{Methods}

Nine hundred seventy-eight healthy individuals who presented to the Gastroenterology Department of Demiroglu Bilim University Faculty of Medicine as liver donor candidates between January 2004 and January 2019 were reviewed retrospectively. Two hundred sixty-nine live liver donor candidates who underwent liver biopsy for various reasons were identified, and biopsy reports were reviewed retrospectively. Forty-one patients were excluded from the study for various reasons (viral hepatitis B carriers, systemic disease, chronic drug usage, patients with inaccessible CT images, etc.). A total of 228 patients with no systemic disease who underwent liver biopsy were identified and included in the study. The demographic, laboratory and abdominal CT scan findings of the patients were retrospectively reviewed and recorded from the hospital central information system. A CT scan was used for the measurement of both HS and PS. All patients included in the study underwent a non-contrast upper abdomen and contrast triphasic CT imaging protocol for the evaluation of the liver with a 16-detector multidetector computed tomography (MDCT) device (Somatom Sensation-Siemens Medical Systems, Forchheim, Germany). 


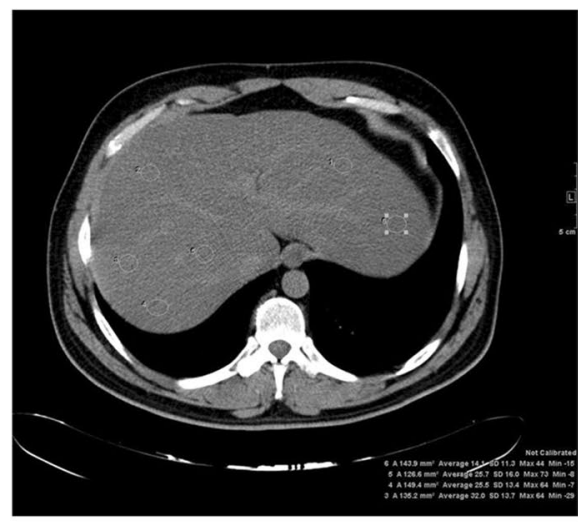

a

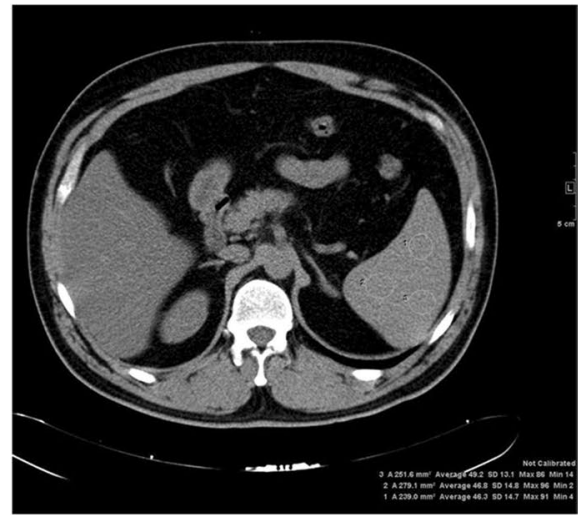

b

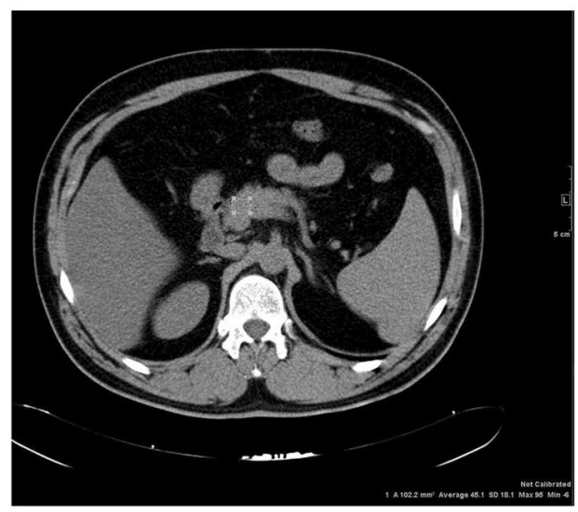

C

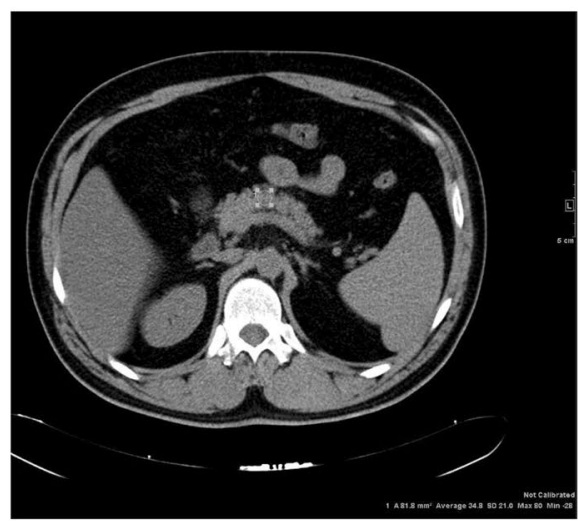

d

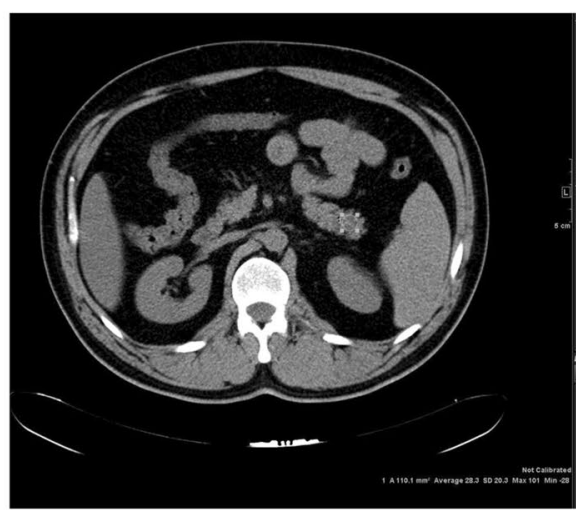

e

Figure 1. Images of a 35-year-old male who is a candidate for liver donor with hepatostaetosis. (a-e) Unhanced transverse CT images are shown. HU was measured with ROI from liver, pancreatic head, body, tail and spleen. Mean pancreatic and splenic CT attenuation was 37 and $47 \mathrm{HU}$, respectively. Difference between pancreatic and splenic attenuation (P-S) and pancreas-to-spleen attenuation ratio (P/S) were - $10 \mathrm{HU}$ and 0.79 . Pancreatic steatosis grade 2 was found according to the parameters used. ( $\left.\mathrm{P}_{\text {mean }}, \mathrm{P}-\mathrm{S}, \mathrm{P} / \mathrm{S}\right)$. The liver attenuation index was found $<-10$ and was accepted as steatosis $>30 \%$.

The liver attenuation index (LAI) was used to calculate the level of HS in CT. Density measurements were performed on an average of 20 regions of interest (ROIs) in the liver and 10 ROIs in the spleen for the evaluation of HS. Areas away from the vessels were selected for density measurements in both organs. The LAI was calculated by subtracting the mean splenic density from the mean hepatic density. LAI $>5$ was accepted as steatosis $<5 \%, 5>\mathrm{LAI}>-10$ was accepted as steatosis between 5 and $30 \%$ and LAI $<-10$ was accepted as steatosis $>30 \%{ }^{37}$ (Fig. 1).

Non-contrast abdominal CT sections taken with a 16-detector MDCT device (Somatom Sensation-Siemens Medical Systems, Forchheim, Germany) were evaluated for the measurement of PS. For the quantitative assessment of the average fat content of the pancreas, the ROIs were selected from the head, trunk and tail sections of the pancreas, each having an area of approximately $1.0 \mathrm{~cm}^{2}$. The Hounsfield Unit (HU) values were measured 
and averaged from the spleen parenchyma in 3 different regions to normalize PS. Artefacts and vascular structures were excluded in CT images, and the peripheral margin of the pancreas was avoided because of the partial volume effect. Pancreatic mean HU value, HU value difference between pancreas and spleen (P-S) and pancreas and spleen $\mathrm{HU}$ value ratio (P/S) were the 3 parameters used to evaluate PS in the study (Fig. 1). Pancreatic fat staging was performed according to these parameters ${ }^{10,18}$. We divided the subjects into three groups according to the degree of each pancreatic steatosis parameter. In our study, our cut-off values for grade 1 pancreatic steatosis were 41.67, -6.33 and 0.87 for Pmean, P-Smean and P/Smean, respectively. In our study, our cut-off values for grade 2 pancreatic steatosis were 36.67, -12 and 0.76 for Pmean, P-Smean and P/Smean, respectively. In our study, our cut-off values for grade 3 pancreatic steatosis were $26.5,-22$ and 0.56 for Pmean, P-Smean and $\mathrm{P} / \mathrm{Smean}$, respectively. Patients with chronic disease who were taking medication and did not have CT or laboratory findings were excluded from the study.

This study was conducted according to the guidelines laid down in the Declaration of Helsinki, and all procedures involving human subjects were approved by the Demiroglu Science University Ethics Committee (approval number 2019-16-03; approved on 08.06.2019). Informed consent was obtained from all participants.

Statistical analysis. SPSS 21.0 for Windows was used for statistical analysis. Descriptive statistics: The number and percentage for categorical variables, mean, standard deviation, minimum, maximum for numerical variables were given. The relationship between numerical variables was examined by Spearman Correlation Analysis since no parametric test condition was provided. The Mantel Haenszel chi-square test was used to investigate the relationships between the groups. The agreement of the evaluations was given by the kappa coefficient. Statistical significance was indicated by $\mathrm{p}<0.05$.

Received: 9 December 2019; Accepted: 9 February 2021

Published online: 24 February 2021

\section{References}

1. Ng, M. et al. Global, regional, and national prevalence of overweight and obesity in children and adults during 1980-2013: A systematic analysis for the global burden of disease study 2013. Lancet 384, 766-781 (2014).

2. Swinburn, B. A. et al. The global obesity pandemic: Shaped by global drivers and local environments. Lancet 378, 804-814 (2011).

3. Reaven, G. M. Banting lecture 1988. Role of insulin resistance in human disease. Diabetes 37, 1595-1607 (1988).

4. Neuman, M. G., Cohen, L. B. \& Nanau, R. M. Biomarkers in nonalcoholic fatty liver disease. Can. J. Gastroenterol. Hepatol. 28, 607-618 (2014).

5. Nascimbeni, F. et al. From NAFLD in clinical practice to answers from guidelines. J. Hepatol. 59, 859-871 (2013).

6. Smits, M. M. \& van Geenen, E. J. The clinical significance of pancreatic steatosis. Nat. Rev. Gastroenterol. Hepatol. 8, 169-177 (2011).

7. Olsen, T. S. Lipomatosis of the pancreas in autopsy material and its relation to age and overweight. Acta. Pathol. Microbiol. Scand. A 86A, 367-373 (1978).

8. Stamm, B. H. Incidence and diagnostic significance of minor pathologic changes in the adult pancreas at autopsy: A systematic study of 112 autopsies in patients without known pancreatic disease. Hum. Pathol. 15, 677-683 (1984).

9. van Geenen, E. J. et al. Nonalcoholic fatty liver disease is related to nonalcoholic fatty pancreas disease. Pancreas 39, 1185-1190 (2010).

10. Jeong, J. S. et al. The association between pancreatic steatosis and diabetic retinopathy in type 2 diabetes mellitus patients. Diabetes Metab. J. 42, 425-432 (2018).

11. Kim, M. K. et al. The association between ectopic fat in the pancreas and subclinical atherosclerosis in type 2 diabetes. Diabetes Res. Clin. Pract. 106(3), 590-596 (2014).

12. Pinte, L., Balaban, D. V., Baicus, C. \& Jinga, M. Non-alcoholic fatty pancreas disease-Practices for clinicians. Rom. J. Intern. Med. 57(3), 209-219 (2019).

13. Bi, Y., Wang, J. L., Li, M. L., Zhou, J. \& Sun, X. L. The association between pancreas steatosis and metabolic syndrome: A systematic review and meta-analysis. Diabetes Metab. Res. Rev. 35, e3142 (2019).

14. Catanzaro, R., Cuffari, B., Italia, A. \& Marotta, F. Exploring the metabolic syndrome: Nonalcoholic fatty pancreas disease. World J. Gastroenterol. 22, 7660-7675 (2016).

15. Kim, S. Y. et al. Quantitative assessment of pancreatic fat by using unenhanced CT: Pathologic correlation and clinical implications. Radiology 271, 104-112 (2014).

16. Zheng, D. et al. Accuracy of computed tomography for detecting hepatic steatosis in donors for liver transplantation: A metaanalysis. Clin. Transplant. 31, e13013 (2017).

17. Bravo, A. A., Sheth, S. G. \& Chopra, S. Liver biopsy. N. Engl. J. Med. 344, 495-500 (2001).

18. Lee, J. S. et al. Clinical implications of fatty pancreas: Correlations between fatty pancreas and metabolic syndrome. World J. Gastroenterol. 15, 1869-1875 (2009).

19. Sepe, P. S. et al. A prospective evaluation of fatty pancreas by using EUS. Gastrointest. Endosc. 73, 987-993 (2011).

20. Singh, R. G. et al. Ectopic fat accumulation in the pancreas and its clinical relevance: A systematic review, meta-analysis, and meta-regression. Metabolism 69, 1-13 (2017).

21. Lesmana, C. R., Pakasi, L. S., Inggriani, S., Aidawati, M. L. \& Lesmana, L. A. Prevalence of non-alcoholic fatty pancreas disease (NAFPD) and its risk factors among adult medical check-up patients in a private hospital: A large cross sectional study. BMC Gastroenterol. 15, 174 (2015).

22. Mathur, A. et al. Nonalcoholic fatty pancreas disease. HPB (Oxford) 9, 312-318 (2007).

23. Pinnick, K. E. et al. Pancreatic ectopic fat is characterized by adipocyte infiltration and altered lipid composition. Obesity 16, 522-530 (2008).

24. Shah, N., Rocha, J. P., Bhutiani, N. \& Endashaw, O. Nonalcoholic fatty pancreas disease. Nutr. Clin. Pract. 34, S49-S56 (2019).

25. Al-Haddad, M. et al. Risk factors for hyperechogenic pancreas on endoscopic ultrasound: A case-control study. Pancreas 38, 672-675 (2009).

26. Choi, C. W. et al. Associated factors for a hyperechogenic pancreas on endoscopic ultrasound. World J. Gastroenterol. 16, 4329-4334 (2010).

27. Wong, V. W. et al. Fatty pancreas, insulin resistance, and beta-cell function: A population study using fat-water magnetic resonance imaging. Am. J. Gastroenterol. 109, 589-597 (2014).

28. Yoon, J. H. et al. Pancreatic steatosis and fibrosis: Quantitative assessment with preoperative multiparametric MR imaging. Radiology 279, 140-150 (2016). 
29. Hu, H. H., Kim, H. W., Nayak, K. S. \& Goran, M. I. Comparison of fat-water MRI and single-voxel MRS in the assessment of hepatic and pancreatic fat fractions in humans. Obesity 18, 841-847 (2010).

30. Tariq, H., Nayudu, S., Akella, S., Glandt, M. \& Chilimuri, S. Non-alcoholic fatty pancreatic disease: A review of literature. Gastroenterol. Res. 9, 87-91 (2016).

31. Heni, M. et al. Pancreatic fat is negatively associated with insulin secretion in individuals with impaired fasting glucose and/or impaired glucose tolerance: A nuclear magnetic resonance study. Diabetes Metab. Res. Rev. 26, 200-205 (2010).

32. Schwenzer, N. F. et al. Quantification of pancreatic lipomatosis and liver steatosis by MRI: Comparison of in/opposed-phase and spectral-spatial excitation techniques. Investig. Radiol. 43, 330-337 (2008).

33. Sijens, P. E., Edens, M. A., Bakker, S. J. \& Stolk, R. P. MRI-determined fat content of human liver, pancreas and kidney. World J. Gastroenterol. 16, 1993-1998 (2010).

34. Hannukainen, J. C. et al. Liver and pancreatic fat content and metabolism in healthy monozygotic twins with discordant physical activity. J. Hepatol. 54, 545-552 (2011).

35. Saisho, Y. et al. Pancreas volumes in humans from birth to age one hundred taking into account sex, obesity, and presence of type-2 diabetes. Clin. Anat. 20, 933-942 (2007).

36. Matsumoto, S. et al. Uneven fatty replacement of the pancreas: Evaluation with CT. Radiology 194, 453-458 (1995).

37. Limanond, P. et al. Macrovesicular hepatic steatosis in living related liver donors: Correlation between CT and histologic findings. Radiology 230, 276-280 (2004).

\section{Acknowledgements}

This research did not receive any specific grant from funding agencies in the public, commercial, or not-forprofit sectors.

\section{Author contributions}

S.T., K.S.B. and O.A. designed the experiments. S.T., K.S.B., O.A. and K.E. contributed to the data collection. S.T. and O.A. conducted the data analysis. S.T., K.S.B., O.A., K.E and I.N. wrote the manuscript. All authors reviewed and approved the final submitted manuscript.

\section{Competing interests}

The authors declare no competing interests.

\section{Additional information}

Correspondence and requests for materials should be addressed to B.K.S.

Reprints and permissions information is available at www.nature.com/reprints.

Publisher's note Springer Nature remains neutral with regard to jurisdictional claims in published maps and institutional affiliations.

(c) (i) Open Access This article is licensed under a Creative Commons Attribution 4.0 International License, which permits use, sharing, adaptation, distribution and reproduction in any medium or format, as long as you give appropriate credit to the original author(s) and the source, provide a link to the Creative Commons licence, and indicate if changes were made. The images or other third party material in this article are included in the article's Creative Commons licence, unless indicated otherwise in a credit line to the material. If material is not included in the article's Creative Commons licence and your intended use is not permitted by statutory regulation or exceeds the permitted use, you will need to obtain permission directly from the copyright holder. To view a copy of this licence, visit http://creativecommons.org/licenses/by/4.0/.

(C) The Author(s) 2021 